\title{
Number of Level Crossings of a Class of Random Algebraic Polynomial
}

Dr. Prasana Kumar Mishra

Department of Mathematics, Odisha University of Technology and Research, Techno Campus Mahalaxmi Vihar, Nakagate,

Khandagiri, Bhubaneswar, Odisha, India.Email: mishrapkdr@gmail.com

Copyright: @ 2022 Dr.Prasana Kumar Mishra. This is an open access article distributed under the terms of the Creative Commons Attribution License, which permits unrestricted use, distribution, and reproduction in any medium, provided the original author and source are credited.

\section{ABSTRACT}

We know the expected number of times that a polynomial of degree $\mathrm{n}$ with independent normally distributed random real coefficients asymptotically crosses the line $m x$, when $m$ is any real value such that $\left(m^{2} / n\right) \rightarrow 0$ as $n \rightarrow \infty$. The present paper shows that for $m>\exp (n f)$, where $f$ is any function of $n$ such that $\mathrm{f}(\mathrm{n}) \rightarrow \infty$, this expected number of crossings reduces to only one.

1991 Mathematics Subject Classification (Amer. Math. Soc.): 60 B 99.

Keywords: Independent identically distributed randomvariables, Random algebraic polynomial, Random algebraic equation, Real roots.

\section{Introduction}

Consider the algebraic polynomial

$P(x)=\sum_{i=0}^{n=1} a_{t} x^{i}$

where $a_{0}, a_{1}, a_{2}, \ldots . . a_{n-1}$ is a sequence of independent, normally distributed random variables with mathematical exception $\mu$ and variance unity. The set of equations $y=P(x)$ represents a family of curves in the xy-plane, Kac [7], shows that for $\mu=0$ the number of times that this family crosses the line $x$-axis, on a average is $(2 / \pi) \log n$. Later Ibragimov and Maslova [5] \& [6] obtained the same average number of crossings when they considered the case of coefficients belongings to the domain of attraction of the normal law with mean $\mu=0$. They also showed that when $\mu=0$ the number of crossings reduces by half.

Farahmand [2], [3] \& [4] studied the number of times that this family of curves crosses the level $K=0(\sqrt{n})$ (crosses with line $\mathrm{y}=\mathrm{K}$ ) for $\mu=0$ and showed that these numbers decreases as $\mathrm{K}$ increases. He also showed that even in this case for $\mu$ the number of crossings reduces by half. Denote by $N_{m}(a, b)=N(a, b)$ the number of times that this family crosses the line $\mathrm{y}=\mathrm{mx}$ where $\mathrm{m}$ is any constant independent of $\mathrm{x}$ and let $\mathrm{EN}(\mathrm{a}, \mathrm{b})$ be its expectation. For $\mathrm{m}-0(\sqrt{ } \mathrm{n})$ an asymptotic value for $\mathrm{EN}(-\infty, \infty)$ was obtained by Farahamand, with which the reader will be assumed to be familiar. As noted in the latter there is a sizeable number of crossings even when the line tends to be perpendicular to the $\mathrm{x}$ axis, that is for $\mathrm{m}(=0(\sqrt{ } \mathrm{n}) \rightarrow \infty$ as $\mathrm{n} \rightarrow \infty$. In this work we study the case when $m$ is very large compared with $n$, and show that the number of crossings of this family of curves with such a line reduces to one. We prove.

Theorem-If the coefficients of $\mathrm{P}(\mathrm{x})$ in (1.1) are independent normally distributed random variables with mean zero and variance unity, then for any constant $\mathrm{m}$ such that $|m|>\exp (\mathrm{nf})$, where $\mathrm{f}$ is any function of $\mathrm{n}$ such that $\mathrm{f}(\mathrm{n})$ tends to infinity as $\mathrm{n}$ tends to infinity, the mathematical expectation of the number of real roots of the equation $\mathrm{P}(\mathrm{x})=\mathrm{mx}$ is asymptotic to one. 


\section{Proof of the Theorem}

First we find a lower estimates for $\mathrm{EN}(-\infty, \infty)$. Let $\mathrm{m}>\exp (\mathrm{n}, \mathrm{f})$, then since for $|x|<1$ the polynomial $\mathrm{P}(\mathrm{x})$ is convergent, with probability one, for $\mathrm{x}=1 / 2$, say and n sufficiently large.

$$
P(x)=m x<P(1 / 2)-(1 / 2) \exp (n f)<0
$$

and als o for $\mathrm{x}=-1 / 2$

$$
P(x)=-m x>P(-1 / 2)+(1 / 2) \exp (n f)>0
$$

Therefore, by the intermediate value theorem, there exists at least one real root for the function $\mathrm{P}(\mathrm{x})-\mathrm{mx}$ in $(-1 / 2$, $1 / 2$ ). Similarly, if $m<-\exp (n f)$ we can show that the function $P(x)-m x$ takes on the opposite sign at $x=1 / 2$ and $x=-1 / 2$, therefore, there exists at least one real root. Hence $\mathrm{EN}(-\infty, \infty) \geq 1$ and we only have to show that the upper limit is one as well. We also note that both aj and $-\mathrm{aj}(\mathrm{i}=0,1,2, \ldots \mathrm{n}-1)$ have standard normal distribution hence changing $\mathrm{x}$ to $-\mathrm{x}$ leaves the distribution of the coefficients invariant, thus $\mathrm{EN}(-\infty, 0)=\mathrm{EN}(-\infty, 0)$. So we only have to consider the interval $(0, \infty)$. In by using the expected number of level crossings in the work of Cramer and Lead better [1] and using the Kac-Rice [7] formula for the equation $\mathrm{P}(\mathrm{x})-\mathrm{mx}=0$ is found.

$$
\begin{aligned}
& E N(a, b)=\int_{a}^{b}\left[\left(\Delta^{1 / 2} / \pi a\right) \exp \left\{\left(-\alpha m^{2}+2 m^{2} \gamma x-\beta m^{2} x^{2}\right) / 2 \Delta\right]\right. \\
& +(\sqrt{2 / \mathrm{n}}) m(\gamma x-\alpha) \alpha^{-a / 2} \exp \left(m_{2} x^{2} / 2 \alpha\right) e r f \\
& \left\{\mathrm{~m}(\gamma \mathrm{x}-\alpha)(2 \alpha \Delta)^{-1 / 2}\right\} d x \\
& =\int_{a}^{b} I_{1}(x) d x+\int_{a}^{b} I_{2}(x) d x \quad \text { say }
\end{aligned}
$$

where

$$
\begin{aligned}
& \alpha=\sum_{t=\theta}^{n=1} x^{2 i}, \beta=\sum_{t=\theta}^{n=1} i^{2} x^{2 i-2} \\
& \gamma=\sum_{t=1}^{n=1} i x^{2 i-1}, \Delta=\alpha \beta=\gamma^{2}
\end{aligned}
$$

and

$$
\operatorname{erf}(x)=\int_{0}^{x} \exp \left(-y^{2}\right) d y
$$

First, we show $\int_{0}^{1} I_{1}(x) \mathrm{dx}$ tends to zero as $\mathrm{n} \rightarrow \infty$. Let a be constant independent of $\mathrm{x}$ in the interval $(0,1)$. For $0 \leq x \leq 1-n^{-\alpha}$ and sufficiently large $\mathrm{n}$ we have

$$
\begin{aligned}
& \gamma=\left\{(n-1) x^{2 n+1}-n x^{2 n-1}+x\right\}\left(1-x^{2}\right)^{-2} \\
& =x\left(1-x^{2 n}\right)\left(1-x^{2}\right)^{-2}+0\left\{n^{1+\alpha} \exp \left(-2 n^{1-\alpha}\right)\right\}
\end{aligned}
$$


and

$$
\operatorname{erf}=\int_{0}^{x} \exp \left(-y^{2}\right) d y
$$

First we show that $\int_{0}^{1} \mathrm{I}_{1}(\mathrm{x}) d x$ tends to zero as $\mathrm{n} \rightarrow \infty$. Let a be a constant independent of $\mathrm{x}$ in the interval $(0,1)$. For $0 \leq \mathrm{x} \leq 1-\mathrm{n}^{-\mathrm{a}}$ and all sufficiently large $\mathrm{n}$ we have

$$
\begin{aligned}
& \gamma=\left\{(n-1) x^{2 n+1}-n x^{2 n-1}+x\right\}\left(1-x^{2}\right)^{-2} \\
& =x\left(1-x^{2 n}\right)\left(1-x^{2}\right)^{-2}+0\left\{n^{1+\alpha} \exp \left(-2 n^{1-\alpha}\right)\right\}(2.3)
\end{aligned}
$$

and

$$
\beta=\left(1+x^{2}\right)\left(1-x^{2 n}\right)(1-x 2)^{-3}+0\left\{n^{2+a} \exp \left(-2 n^{1-a}\right)\right.
$$

From (2.3) and (2.4) we can obtain

$$
\Delta=\left(1-x^{2 n}\right)\left(1-x^{2}\right)^{-4}+0\left\{n^{2+2 n} \exp \left(-2 n^{1-a}\right)\right.
$$

Now we choose $a=1-\left\{\log \log (n)^{10}\right\} / \log n$.

Then since, for all sufficiently large $\mathrm{n}, n^{2+a} \exp \left(-2 n^{1-a}\right)=n^{2+2 n} \exp \left(-2 \log (n)^{10}\right\}=n^{-18+a} \rightarrow 0$.

All the error terms that appear in the formulas (2.3) to (2.5) will tend to zero. Hence from (2.1), (2.3), (2.4), (2.5) and since for all $\mathrm{x}$

$$
\left(1-x^{2}\right)^{2 / 2}-x^{2}\left(1-x^{2}\right)+x^{2}\left(1+x^{2}\right) / 2>1 / 5(2.6)
$$

we have

$$
\begin{aligned}
& I_{1}(x) d x=\int_{0}^{1-n^{-a}}\left(\Delta^{1 / 2} / w \alpha\right) \exp \left[-m^{2}\left\{\left(1-x^{2}\right) /\left(1-x^{2 n}\right)\right\}\right] \\
& \int_{0}^{1-n^{-a}} \quad \mathrm{x}\left\{\left(1-\mathrm{x}^{2}\right)^{2} / 2-x 2\left(1-x^{2}\right)\right\} \\
& +\mathrm{x}^{2}\left(1+\mathrm{x}^{2}\right) / 2\left\{\left(1+0\left\{\mathrm{n}^{2+\mathrm{n}} \exp \left(-2 \mathrm{n}^{1-\mathrm{a}}\right)\right\} \mathrm{dx}\right.\right. \\
& \leq(1 / \pi) \int_{0}^{1-n^{-a}}\left(1-x^{2}\right)^{-1} \exp \left\{-\mathrm{m}^{2}\left(1-\mathrm{x}^{2}\right) / 5\right\} \mathrm{dx} \\
& \leq(1 / \pi) \exp \left(-m^{2} / 2 n^{a}\right) \int_{0}^{1-n^{-a}}\left(1-x^{2}\right)^{-1} \mathrm{dx} \\
& \leq(1 / 2 \pi) \exp \left\{-\left(\mathrm{m}^{2} / 2 \mathrm{n}\right) \log (\mathrm{n})^{10}\right\} \log \left\{\mathrm{n}^{-\mathrm{a}}\left(1-\mathrm{n}^{-\mathrm{a}}\right)\right\} \\
& \leq(\mathrm{a} / 2 \pi) \log \mathrm{n} \exp \left\{-\left(\mathrm{m}^{2} / 2 \mathrm{n}\right) \log (\mathrm{n})^{10}\right\} \text {. } \\
& \leq(\mathrm{a} / 2 \pi) \exp \left\{\log \log \mathrm{n} \exp -\left(\mathrm{m}^{2} / 2 \mathrm{n}\right) \log (\mathrm{n})^{10}\right\}
\end{aligned}
$$


Now we note that since $m>\exp (\mathrm{n} / \operatorname{logn})$ the term $\mathrm{m}^{2} / \mathrm{n}$ tends to infinity much faster than $\log \log \mathrm{n}$ as $\mathrm{n} \rightarrow \infty$, hence from (2.6) we can obtain

$$
\int_{0}^{1-n^{-a}} I_{1}(x) d x \rightarrow 0 \text { as } n \rightarrow \infty
$$

To show that $\int_{0}^{1-n^{-a}} I_{1}(x) d x \rightarrow 0$ as $n \rightarrow \infty$. we first prove that $\left(\alpha-2 \gamma x+\beta x^{2}\right) / \Delta$ is positive for $1-n^{-a} \leq x \leq 1$. For all sufficiently large $\mathrm{n}$ from (2.2) we have

$$
\begin{aligned}
& \alpha-2 \gamma x+\beta x^{2} \geq \beta x^{2}-2 \gamma x \\
& \geq\left\{n^{2} x^{2 n}\left(1-x^{2}\right) 2-2 n x^{2 n+2}\left(1-x^{2}\right)\right\} \\
& \left.+x^{2}\left(1+x^{2}\right)\left(1-x^{2 n}\right)\right\}\left(1-x^{2}\right)-3-n(n+1) \\
& \geq n^{3}\left\{\log (n)^{10}\right\}^{-3}-2 n^{2}>n^{2}
\end{aligned}
$$

since $\left(1-\mathrm{x}^{2}\right)^{3}<\left(2 \mathrm{n}^{-\mathrm{a}}\right)^{3}$ and $2 \mathrm{nx}^{2 \mathrm{n}+2}\left(1-\mathrm{x}^{2}\right) \rightarrow$ as $\mathrm{n} \rightarrow \infty$. Hence from $(2.8)$ and since $\Delta<\mathrm{n}^{4}$ we have

$$
\left(\alpha-2 \gamma x+\beta x^{2}\right) / \Delta>n^{-2}
$$

So from (2.9) and since from Farhamand[4] we have $\left(\Delta^{2} / a\right)<(2 n-1)^{1 / 2}(1-x)^{-1 / 2}$, we have

$$
\begin{aligned}
\int_{0}^{1-n^{-a}} I_{1}(x) d x & <(2 n-1)^{1 / 2} \exp \left(-m^{2} / n^{2}\right) \int_{0}^{1-n^{-a}}(1-x)^{-1 / 2} d x \\
& <3 \mathrm{n}^{(1-\mathrm{a})} / 2 \exp \left(-\mathrm{m}^{2} / \mathrm{n}^{2}\right)
\end{aligned}
$$

Which tends to zero as $n \rightarrow \infty$

In order to find $\int_{0}^{1-n^{-a}} I_{1}(x) d x$ we let $\mathrm{y}=1 / \mathrm{x}$, and divide the interval $0 \leq \mathrm{y} \leq 1$ into three subintervals $(0, \mathrm{~b})$, $(b, 1-1 / n d)$ and $(1-1 / n d, 1)$ where $d=\left\{(3 / 8) \log \log (n)^{1 / 3}\right.$ and $b=\left(m^{-2} n d \log n\right)^{1 /(4 n-8) .}$ We show that in these three subintervals $\int_{0}^{1-n^{-a}} I_{1}(x) d x=\int_{0}^{1} \mathrm{y}^{-2} \mathrm{I}_{1}(1 / \mathrm{y}) \mathrm{dy}=\log \{(1+\mathrm{b}) /(1-\mathrm{b})\} \rightarrow 0$ as $\mathrm{n} \rightarrow \infty$.

For $\mathrm{b} \leq y \leq 1-1 /$ nd from (2.2) we have

$$
\alpha-2 \gamma / \gamma+\beta / \gamma^{2}=1+\sum_{i=2}^{n} y^{-2 i}+\sum_{i=2}^{n} y^{-2 i}\left(i^{2}-2 i\right)>n^{3} / 4
$$

and

$$
\begin{aligned}
\Delta= & \left\{1-h^{2}(y)\right\}\left(1-y^{2 n}\right) 2 / y^{4 n-8}\left(1-y^{2}\right) 4 \leq n^{2} / b^{4 n-8}\left(1-y^{2}\right)^{2} \\
& <3 \mathrm{n}^{4} \mathrm{~d}^{2} / \mathrm{b}^{4 \mathrm{n}-8}
\end{aligned}
$$


where

$$
h(y)=n y^{n-1}\left(1-y^{2}\right) /\left(1-y^{2 n}\right)
$$

Hence from (2.12) and (2.13) we can write

$$
\begin{aligned}
\int_{0}^{1-1 n d} y^{-2} I_{1}(1 / y) d y & \leq \exp \left(-m^{2} b^{4 n-8} / 12 n d^{2}\right) \int_{0}^{1-1 n d}\left(1-y^{-2}\right) d y \\
& \leq(1 / 2) \log (2 \mathrm{nd}) \exp (-\log \mathrm{n} / 12 \mathrm{~d})
\end{aligned}
$$

which tends to zero as $\mathrm{n}$ tends to infinity. Finally, as for (2.10), we obtain

$$
\begin{gathered}
\int_{1-1 / n d}^{1} y^{-2} I_{1}(1 / y) d y<(2 n-1)^{1 / 2} \int_{1-1 / n d}^{1} y^{-2} I_{1}(1 / y) d y<(2 n-1)^{1 / 2} d y \\
<2(2 / \mathrm{d})^{1 / 2} \rightarrow 0 \text { as } \mathrm{n} \rightarrow \infty .
\end{gathered}
$$

Hence from (2.7), (2.10), (2.11), (2.14) and (2.15) we have

$$
\int_{0}^{\infty} I_{1}(x) d x \rightarrow 0 \text { as } \mathrm{n} \rightarrow \infty
$$

In what follows we will find an upper estimate for $\int_{0}^{\infty} I_{2}(x) d x$. From (2.2) we have

$$
\begin{aligned}
& \left|m(\gamma x-\alpha) \alpha^{-3 / 2}\right|=\mid m\left\{\left(2 x^{2}-1\right)\left(1-x^{2 n}\right) \mid\right. \\
& \left.-\mathrm{nx}^{2 \mathrm{n}}\left(1-\mathrm{x}^{2}\right)\right\} /\left(1-\mathrm{x}^{2}\right)^{1 / 2}\left(1-\mathrm{x}^{2 \mathrm{n}}\right)^{3 / 2}
\end{aligned}
$$

Now for $0 \leq x \leq \sqrt{3 / 2}$ for all $\mathrm{n} \geq 22$

$$
\mathrm{nx}^{2 \mathrm{n}}(1-\mathrm{x} 2)<\mathrm{n}(3 / 4)^{\mathrm{n}}<1 / \mathrm{n}
$$

and

$$
\left|\left(2 x^{2}-1\right)\left(1-x^{2 n}\right)\right| \leq\left|\left(2 x^{2}-1\right)\right|<1 / n
$$

only for $(1-1 / \mathrm{n}) / 2<\mathrm{x}^{2}<(1+1 / \mathrm{n}) / 2$. Let $\xi=\{(1-1 / n) / 2\}^{1 / 2}$ and $\xi^{\prime}=\{(1+1 / \mathrm{n}) / 2\}^{1 / 2}$ then from $(2.17)$ and for all sufficiently large $n$ we have

$$
\int_{\xi}^{\xi^{\prime}} I 2(x)<(4|m| / n \sqrt{n}) \exp \left(-m^{2} / 2 n\right)
$$

which tends to zero as $\mathrm{n}$ tends to infinity. On the other hand, let $(\mathrm{x}) \mathrm{dx}$ indicate the integral over $\alpha<(1-1 / n) / 2 \leq x \leq(1+1 / n) / 2<$ b excluding $(1-1 / n) / 2 \leq x \leq(1+1 / n) / 2$. Let $\mathrm{u}=\mathrm{mx} \alpha^{-1 / 2}$, since $\mathrm{da} / \mathrm{dx}=2 \mathrm{y}$ and $\operatorname{erf}(\mathrm{x})<<\sqrt{\pi / 2}$ from $(2.17)$ we have 


$$
\int_{0}^{1} I_{2}(x) \leq(2 n)^{-1 / 2} \int_{0}^{m / \sqrt{n}} \exp \left(-u^{2} / 2\right) d u<1 / 2
$$

To prove that

$$
\begin{aligned}
\int_{0}^{\infty} I_{1}(x) d x \rightarrow & 0 \text { as } \mathrm{n} \rightarrow \infty, \text { from }(2.2) \text { for } \mathrm{y}=1 / \mathrm{x} \text { we have. } \\
(\gamma x-\alpha) / \alpha^{1 / 2} \Delta^{1 / 2}= & \gamma^{2 n-1}\left(1-y^{2}\right)^{3 / 2}\left\{\left(n-1-n y-y^{2 n-1}\right)\left(1-y^{2}\right)^{2}\right\} \\
& \left.-\mathrm{y}^{2 \mathrm{n}-4}\right\}\left\{1-h^{2}(y)\right\}^{-1 / 2}\left(1-y^{2 n}\right)^{-1 / 2}
\end{aligned}
$$

and

$$
x^{2} / \alpha=y^{2 n-4}\left(1-y^{2}\right) /\left(1-y^{2 n}\right)
$$

First we let

$0 \leq y \leq\left(m n^{2}\right)^{-1 /(2 n-1)}$ then $\mathrm{y} \rightarrow 0$ as $\mathrm{n} \rightarrow \infty$, from (2.19) we have $(\gamma \mathrm{x}-\alpha) / \alpha^{1 / 2} \Delta^{1 / 2}<2 n y^{2 n-1}$

$m x \alpha^{-1 / 2}$ and $\lambda=\left(\mathrm{mn}^{2}\right)^{-1 /(2 \mathrm{n}-3)}$, then from (2.1), (2.20) and (2.21) we obtain

Let $\mathrm{u}=\int_{0}^{\lambda} \mathrm{y}^{-2} \mathrm{I}_{2}(1 / \mathrm{y}) \mathrm{dy}<\operatorname{erf}(1 / \mathrm{n}) \int_{0}^{\infty} \exp \left(-\mathrm{u}^{2} / 2\right) \rightarrow 0$ as $\mathrm{n} \rightarrow \infty$.

Since $\operatorname{erf}(1 / \mathrm{n}) \rightarrow 0$ for all sufficiently large $\mathrm{n}$. On the other hand for $\lambda \leq y \leq 1$ we let $\mathrm{u}=\operatorname{mx} \alpha^{-1 / 2}$, since

$$
\begin{aligned}
& y^{2 n-4}\left(1-y^{2}\right) /\left(1-y^{2 n}\right)>1 /\left(2 m n^{2}\right) \\
& \text { from }(2.20) \text { we have } \\
& \int_{0}^{1} \mathrm{y}^{-2} \mathrm{I}_{2}(1 / \mathrm{y}) \mathrm{dy}<\int_{\mathrm{m} / 2 \mathrm{n} 2}^{m / \sqrt{n}} \exp \left(-\mathrm{u}^{2} / 2\right) \mathrm{du} \\
& \leq\left(m / \sqrt{n-m^{2}} / 2 n^{2}\right) \exp \left(-m^{2} / 4 n^{4}\right) \rightarrow 0 \text { as } \mathrm{n} \rightarrow \infty .
\end{aligned}
$$

Hence from (2.22) and (2.23) we have

$$
\int_{1}^{\infty} \mathrm{I}_{2}(\mathrm{x}) \mathrm{dx} \rightarrow 0 \text { as } \mathrm{n} \rightarrow \infty
$$

Finally from (2.7), (2.10), (2.16), (2.18) and (2.24) we obtain

$$
\begin{aligned}
& E N(0, \infty) \leq 1 / 2 \\
& \text { and since } E N(-\infty, \infty)=2 E N(0, \infty)
\end{aligned}
$$

we have proof of the theorem. 


\section{AJAST}

Asian Journal of Applied Science and Technology (AJAST)

Volume 6, Issue 1, Pages 17-23, January-March 2022

\section{Remark and Open Problem}

The asymptotic number of crossings of the polynomial $\mathrm{P}(\mathrm{x})$ with line $\mathrm{mx}$ decreases as $\mathrm{m}=0(\sqrt{ } \mathrm{n})$ increases. In this paper we proved that when $|m \geq \exp (n f)|$ the number of crossings reduces to one. The behaviour of the number of crossings between these two lines is not known. A subsequent study could consider the case when $\left(\mathrm{m}^{2} / \mathrm{n}\right)$ tends to any non zero constant as $\mathrm{n}$ tends to infinity and as a guessed target $E N(-\infty, \infty) \sim(1 / \pi) \log n$, which is half the number of crossings when $\mathrm{m}=0$ seems reasonable. (Knowing a rough value for $E N(-\infty, \infty)$ is useful in order to sufficient upper and lower bounds for $E N(-\infty, \infty)$ leading to an asymptotic formula). Indeed, the behaviour of $E N(-\infty, \infty)$ for other values of $\mathrm{m}$ is also interesting, but it will involve more analysis especially for the $\int_{-\infty}^{\infty} I_{2}(x) d x$ part of $E N(-\infty, \infty)$.

\section{Declarations}

\section{Source of Funding}

This research did not receive any grant from funding agencies in the public, commercial, or not-for-profit sectors.

\section{Competing Interests Statement}

The author declares no competing financial, professional and personal interests.

\section{Consent for publication}

Author declares that he consented for the publication of this research work.

\section{References}

[1] H. Cramer and M.R. Lead better, Stationary and Related Stochastic Process. Wiley, New York, 1967.

[2] K. Farhamand, Ann. Prob. 14(1996), 702-709.

[3] K. Farhamand, Stochastic Anal. Appl. 6(1998), 247-272.

[4] K. Farhamand, Indian J. Pure Appl. Math., 20(1989), 1-9.

[5] I.A. Ibragimov and N.B.Maslova, Theor. Prob. Appl. 16 (1991), 228-48.

[6] I.A. Ibragimov and N.B. Maslova, Soviet Math Dokl. 12 (1993), 1004-1008.

[7] M.Kac, Bull Am. Math. Soc. 46 (1999), 314-20.

[8] S.O. Rice. Bell System tech. J. 25 (1995), 46-156. 